\title{
Measurement of the state of stress in silicon with micro-Raman spectroscopy
}

\author{
Stephen J. Harris, ${ }^{\text {a) }}$ Ann E. O'Neill, and Wen Yang \\ Physical and Environmental Science Department, Mail Drop 3083 and Ford Research and Advanced \\ Engineering Center, Dearborn, Michigan 48121 \\ Peter Gustafson \\ Analytical Powertrain Department, Ford Research and Advanced Engineering Center, \\ Dearborn, Michigan 48121 \\ James Boileau \\ Materials Research and Advanced Engineering Department, Ford Research and Advanced Engineering \\ Center, Dearborn, Michigan 48121 \\ W. H. Weber \\ Physics Department, University of Michigan, Ann Arbor, Michigan 48109-1120
}

Bhaskar Majumdar

Materials Science and Metallurgical Engineering Department, New Mexico Institute of Technology, Socorro, New Mexico 87801

Somnath Ghosh

Mechanical Engineering Department, Ohio State University, Columbus, Ohio 43210

(Received 3 June 2004; accepted 31 August 2004)

\begin{abstract}
Micro-Raman spectroscopy has been widely used to measure local stresses in silicon and other cubic materials. However, a single (scalar) line position measurement cannot determine the complete stress state unless it has a very simple form such as uniaxial. Previously published micro-Raman strategies designed to determine additional elements of the stress tensor take advantage of the polarization and intensity of the Raman-scattered light, but these strategies have not been validated experimentally. In this work, we test one such stategy [S. Narayanan, S. Kalidindi, and L. Schadler, J. Appl. Phys. 82, 2595 (1997)] for rectangular (110)- and (111)-orientated silicon wafers. The wafers are subjected to a bending stress using a custom-designed apparatus, and the state of (plane) stress is modeled with ABAQUS. The Raman shifts are calculated using previously published values for silicon phonon deformation potentials. The experimentally measured values for $\sigma_{x x}, \sigma_{y y}$, and $\tau_{x y}$ at the silicon surface are in good agreement with those calculated with the ABAQUS model. (C) 2004 American Institute of Physics. [DOI: 10.1063/1.1808244]
\end{abstract}

\section{INTRODUCTION}

The increased use of cast aluminum components has been an important factor in reducing weight in vehicles. With the increased use has come a need to better understand the mechanical properties of the cast aluminum alloys, especially the mechanisms of ductile fracture, which is only partially understood in cast aluminum alloys. It has been proposed that the initial step in the ductile fracture of cast aluminum is the cracking of the eutectic silicon particles. This cracking is believed to arise from the stresses induced in the particles by the plastic deformation of the aluminum matrix. ${ }^{1}$ These cracked particles nucleate microcracks within the aluminum matrix that eventually join together, leading to a critical-sized crack that can quickly propagate to failure.

In order to predict ductile failure, one must understand load transfer to the particles from the matrix, and one must know the states of stress that cause silicon particles to crack. There are several theories that predict how load transfers to particles. $^{2-4}$ However, these theories generally make simpli-

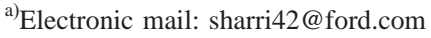

fying assumptions such as that the particles have simple shapes or that the particles are in a homogeneous or periodic environment. These assumptions are not met by the silicon particles typically found in cast aluminum. As shown in Fig. 1 , the silicon particles are complex in shape and are clustered in distribution. Because clustering of silicon particles may play a dominant role in determining ductility ${ }^{1}$ as well as other mechanical properties, ${ }^{5}$ it is critical that its effects on load transfer be well understood. This is not yet the case in cast aluminum alloys. ${ }^{6}$ Experimentally, macroscopic measurements of the far-field strain coupled with load transfer calculations do not allow an accurate determination of the stresses in individual particles. The full state of stress of silicon particles can be measured in situ with neutron diffraction. However, because of the relatively large size of the neutron beam, the measurements are averaged over thousands of particles, including particles with different shapes and environments. This means that these measurements also do not determine the local stress values.

Raman spectroscopy has been widely used to analyze local stresses and strains in a variety of materials and composites, particularly cubic materials such as silicon and sili- 


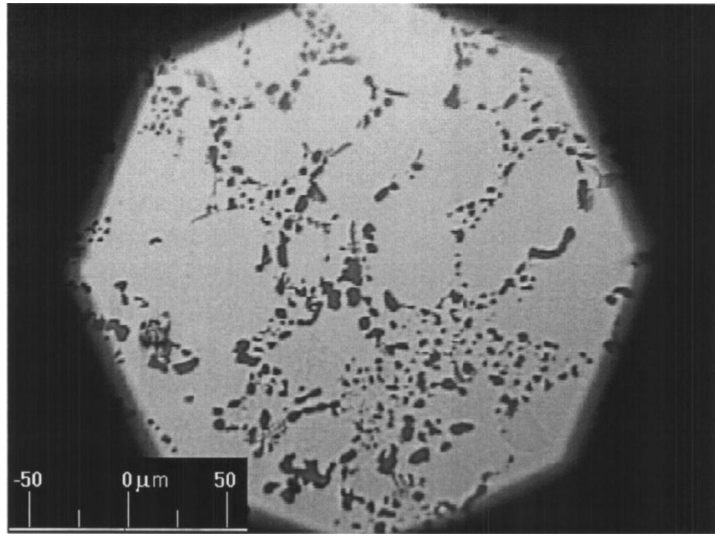

FIG. 1. Optical micrograph of 319 aluminum alloy. The grey bodies are mostly eutectic silicon particles, which range in size from about 1 to $20 \mu \mathrm{m}$.

con carbide. ${ }^{7}$ Typically, the shift in the Raman frequency is calibrated at a known stress, and then this calibration factor is used to determine the stress for the same material in a new situation. For simple states of stress (e.g., uniaxial or equal biaxial), this procedure can be appropriate. But because stress is a tensor quantity with up to three (for plane stress) or six (for the general state) independent components, the stress state cannot, in general, be determined with a single scalar measurement. ${ }^{8}$ In some cases, the elements of the stress tensor can be deduced by measuring the shifts of the individual components of the Raman line. ${ }^{9,10}$ For silicon, however, the stresses are generally not sufficient to give resolved line splittings. In other cases, the state of stress is determined either by a calculation or by a diffraction technique, and then the Raman analysis can be used for scaling and validation. ${ }^{8,11}$

It is possible, however, to get additional information from the Raman measurements because the polarization and intensity of the Raman signal ${ }^{8}$ are influenced by all of the components of the stress tensor. Loechelt et al. ${ }^{12}$ showed that this information can be used to determine the complete stress state, but their proposed experimental geometry is unsuitable for micro-Raman spectroscopy. Recently, Bonera et al. ${ }^{13} \mathrm{de}-$ scribed a strategy for measuring the stress state in a silicon crystal with a (100) orientation, while Narayanan et al. ${ }^{14}$ proposed a strategy for measuring the stress state in a silicon crystal with any orientation except (100). Their approaches are particularly significant because they are compatible with the backscatter geometry typically used for micro-Raman spectroscopy. However, neither group provided experimental verification of their strategy by measuring the state of stress

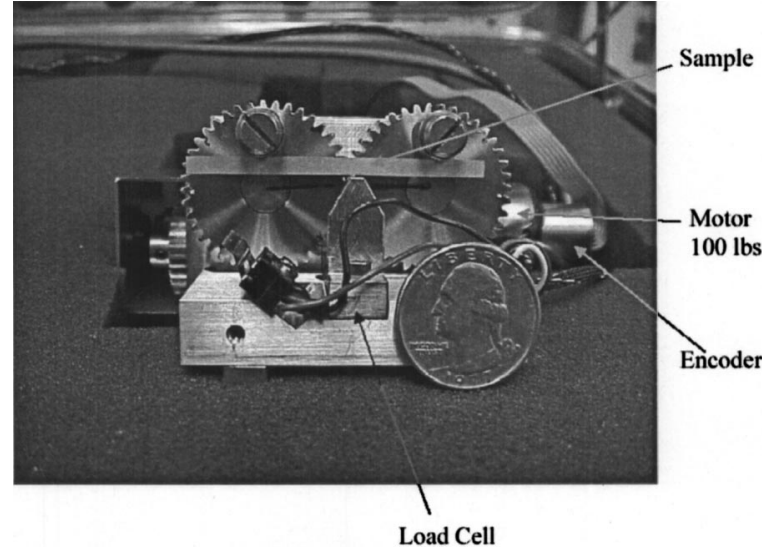

FIG. 2. Mechanical bending device used to strain the silicon wafers. A 3-mm-thick aluminum bar, identified as Sample, is shown in the figure where the silicon wafer would be.

in a silicon sample where the state of the applied stress was already known.

In this work, we describe experiments that validate the strategy of Narayanan et al. ${ }^{14}$ on silicon wafers with several different crystallographic orientations. It is our eventual aim to provide experimental in situ measurements of the stress states of individual silicon particles before and during ductile fracture experiments on cast aluminum. These would include measurements in cracked particles, whose presence can have an important effect on the matrix flow. ${ }^{15}$

\section{EXPERIMENT}

For this study, 100-mm-diameter, 0.38-mm-thick silicon wafers (doping levels near $\left.10^{14} \mathrm{~cm}^{-3}\right)$ were sliced into $60(x$ direction) $\times 4$-mm ( $y$ direction) rectangular wafers. The wafers' crystallographic orientations are given in Table I.

The rectangular wafers were strained using the bending apparatus shown in Fig. 2. The wafers were supported from below by a steel plate that rested on a load cell. Behind the wafer was a pair of gears in mesh, and each gear had a pin with a roller bearing sleeve that rested on the top surface of the wafer. A motor drove the gears, and as the gears rotated, the pins applied a bending force to the silicon wafer over the support, while an encoder measured the angle of rotation $(\theta)$ of the gears. With this arrangement, the force on the wafer from the pins was always normal to the wafer surface at the contact points; almost no tangential force was applied because of the presence of the roller bearings. At the top center of the wafers, the major component of stress was $\sigma_{x x}$, along the 60 -mm length of the wafer.

TABLE I. Crystallographic orientations of the silicon wafers used in this study. The right-most column is the ABAQUS prediction for the ratio of the transverse $(y)$ to longitudinal $(x)$ stress induced by the load cell (see Fig. 2). The wafers are predicted to be in a nearly uniaxial stress.

\begin{tabular}{ccccc}
\hline \hline Wafer \# & Surface normal & Major stress direction $x$ & Laser polarization & $\sigma_{y y} / \sigma_{x x}$ \\
\hline 1 & $(001)$ & $(100)$ & $(010)$ & 0.056 \\
2 & $(001)$ & $(1-10)$ & $(110)$ & 0.043 \\
3 & $(111)$ & $(1-10)$ & $(11-2)$ & 0.047 \\
4 & $(110)$ & $(1-12)$ & $(1-1-1)$ & 0.038 \\
\hline \hline
\end{tabular}


The Raman experiments are carried out with a Renishaw 1000 Raman microscope using the 633-nm line from a $\mathrm{He}-\mathrm{Ne}$ laser. The bending device is oriented so that the incident laser light is polarized along the $y$ direction. The laser is focused at the top center of the wafer through a $20 \times$ objective lens with a $2-\mathrm{cm}$ working distance to give a 2 - to $3-\mu \mathrm{m}$ diameter laser spot. The relatively long focal length helps ensure that the incident and scattering angles are nearly perpendicular to the surface, so that the polarization states are well defined. ${ }^{13}$ Because the optical absorption coefficient of silicon at $633 \mathrm{~nm}$ is approximately 3.9 $\times 10^{3} \mathrm{~cm}^{-1}$, ${ }^{16}$ only the top few microns of the silicon wafer (out of a total thickness of $380 \mu \mathrm{m}$ ) are sampled, a region that we expect to be in a plane stress. The laser power at the focus is approximately $2 \mathrm{~mW}$, corresponding to an average intensity of about $40 \mathrm{~kW} / \mathrm{cm}^{2}$. Because of the relatively small absorption coefficient of silicon at the He-Ne wavelength (compared to that at $\mathrm{Ar}^{+}$wavelengths), the laser power is spread over a relatively large volume, and the estimated temperature rise in the illuminated volume is negligible. The backscattered Raman light is collimated and passed through a rotatable Polaroid analyzer before entering the spectrometer, which has a 1200 -groove/mm grating that produces a dispersion of $1.68 \mathrm{~cm}^{-1} /$ pixel. A charge coupled device (CCD) detects the light. The silicon line near $520 \mathrm{~cm}^{-1}$ is a convolution of its intrinsic shape and the spectrometer instrument function, and its width is about $4 \mathrm{~cm}^{-1}$. The silicon line is fit between 500 and $540 \mathrm{~cm}^{-1}$ using a five-parameter pseudo- Voigt profile,

$$
\begin{aligned}
y(\omega)= & y_{0}+a\left[c\left(\frac{1}{1+\left(\frac{\omega-\omega_{0}}{b}\right)^{2}}\right)\right. \\
& \left.+(1-c) e^{-0.5\left(\omega-\omega_{0} / b\right)^{2}}\right] .
\end{aligned}
$$

Typically, $c$ is close to 0.5 (equal parts Gaussian and Lorentzian), and the $R^{2}$ correlation coefficient for the fit is between 0.9990 and 0.9999 . Although neither the resolution of the spectrometer nor the pixel density of the CCD is particularly high, the repeatability of the spectra is excellent because of the extremely high signal-to-noise ratio $\left(\sim 10^{3}\right)$. As a result, the standard error for the line center position $\omega_{0}$ is quite small, between 0.01 and $0.02 \mathrm{~cm}^{-1}$. The Raman spectra are accumulated for whatever time is required to give $30000-40000$ counts at the peak of the silicon line, typically between 60 and $600 \mathrm{~s}$. In order to account for the drift in the spectrometer, the spectra taken from the stressed wafer are alternated with the measurements taken from an unstressed silicon wafer.

The Polaroid analyzer is slightly wedged, which causes the path of the light entering the spectrometer to be bent by about $0.5 \mathrm{mrad}$. Because this entrance angle affects the frequency measurement of the spectrometer, rotating the Polaroid analyzer by $180^{\circ}$ about its own axis changes the re-
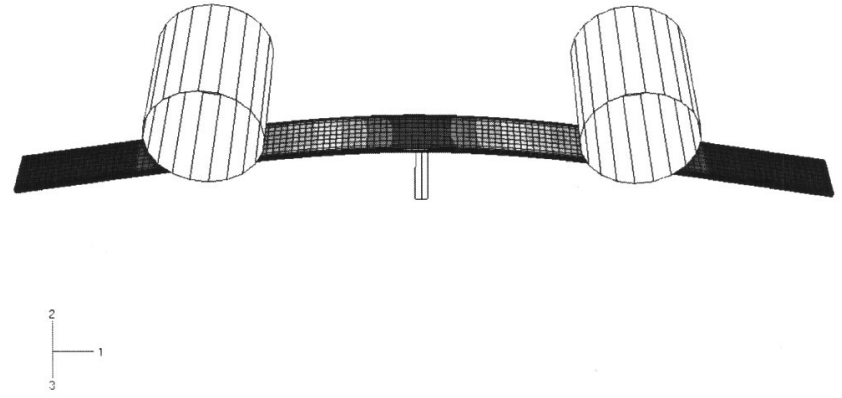

FIG. 3. Schematic view of the finite element ABAQUS model.

sults. To avoid this problem, we place two Polaroid analyzers in the light path, in series, oriented such that the effect is canceled.

\section{ANALYSIS}

Despite the simple appearance of the test setup, the deformed mechanical state of the silicon specimen cannot be analyzed with a conventional beam theory. As a cubic material, the stiffness is dependent on the material orientation and can exhibit coupling among extension, bending, and twisting. Additionally, the out-of-plane depth of the specimen is relatively large (the specimen is ten times wider than it is thick). Consequently, the three-dimensional model shown in Fig. 3 was created in ABAQUS to represent the specimen and bending mechanism.

In the ABAQUS model, the bending mechanism is represented by three rigid surfaces that are displacement controlled. The rigid brick elements represent the support and are fixed in space. Two cylinderical analytical surfaces represent the rollers. All contact is modeled using ABAQUS surface-based contact, with finite sliding. Coulomb friction (0.2) was included in order to improve the solution convergence. (Friction in the range of 0.0-0.2 was found to have a negligible effect on the predicted stress in the areas of interest.) The rollers move in paths that correspond to the arcs traced out by the rollers as each gear rotates. The silicon is represented by hexahedral elements, in which the material properties are assumed to be linear elastic and cubic. Material properties are applied at different orientations in each model. No attempt is made to include silicon oxidation in the model, as it has been shown ${ }^{11}$ that the presence of an oxide layer does not measurably affect the stress in the silicon. A nonlinear geometry is enabled to ensure a robust solution as the specimen deformed. Appropriate constraints are applied to prevent a rigid body motion.

The Raman analysis follows the strategy suggested by Narayanan et al., ${ }^{14}$ who showed that, in principal, all three (plane) stress components of silicon can be determined by using polarization, intensity, and frequency shift information from the three components of the $520-\mathrm{cm}^{-1}$ line. The mathematical details of the analysis are given in their paper ${ }^{14}$ and will only be briefly summarized here. At zero stress, the Raman line in silicon is triply degenerate. When stress is applied, the line will, in general, split into three components having different frequencies, intensities, and polarizations. These frequencies are the eigenvalues of a $3 \times 3$ determinant, 
the input parameters for which are the strain components and the phonon deformation potentials (PDPs). ${ }^{8}$ The eigenfunction for each eigenvalue is also found from the same $3 \times 3$ matrix. The predicted spectrum for a specific scattering geometry and setting of the polarization analyzer can then be determined by applying the standard polarization selection rule,

$$
I=C \sum_{j=1}^{3}\left|\mathbf{e}_{L} \cdot \mathbf{R}_{j} \cdot \mathbf{e}_{s}\right|^{2},
$$

where $\mathbf{e}_{L}$ and $\mathbf{e}_{s}$ are the unit vectors in the laser and scattered-light polarization directions, $\mathbf{R}_{j}$ are the eigenvalues of the individual Raman lines, $C$ is a constant, and $I$ is the measured intensity.

The three eigenvalues are never resolved in the observed Raman spectrum. However, because the polarizations of the three components are different, the relative intensities of the three components at the CCD vary as a function of the Polaroid analyzer angle, which means that the observed center frequency of the Raman line varies. Narayanan et al. suggested that the overall peak shift could be predicted by a simple weighting formula,

$$
\overline{\Delta \omega}=\sum_{i=1}^{3} \frac{\Delta \omega_{i} I_{i}}{I_{T}},
$$

where $\Delta \omega_{i}$ is the peak shift for the $i$ th component of the line, $I_{i}$ is its corresponding intensity, and $I_{T}$ is the sum of the intensities of the three initially degenerate peaks. De Wolf et $a l$. used a similar weighting formula ${ }^{8}$ in their work. Thus, for any state of stress, the analysis predicts the variation in the "sensitivity" (which we define to be the Raman shift per unit of $\sigma_{x x}$ ) as a function of the analyzer angle. As an example, Fig. 4 shows the experimentally measured sensitivity of the Raman line center position to stress when the analyzer is set at $0^{\circ}$ and $90^{\circ}$, measured from the laser polarization, for wafer \#3. The strategy depends on inverting the process, determining the three stress components from a measurement of the line center position versus the analyzer angle. The strategy can be successful if the inversion is unique.

\section{RESULTS AND DISCUSSION}

The strain gauge measurements of $\varepsilon_{x x}$ as a function of gear angle $\theta$ were in good agreement with the predictions of the ABAQUS model, with both the model and the experiment showing a near-linear relationship between $\varepsilon_{x x}$ and $\theta$ in the region of interest. We measured $d \varepsilon_{x x} / d \theta=1.24 \pm 0.07$ $\times 10^{-4}$ degree $^{-1}(1 \sigma)$, compared to the ABAQUS result $d \varepsilon_{x x} / d \theta=1.23 \times 10^{-4}$ degree $^{-1}$, independent of the crystallographic orientation of the silicon wafer. The relationship between $\varepsilon_{x x}$ and $\sigma_{x x}$ is obtained from the ABAQUS model. Table I shows the ABAQUS predictions for $\sigma_{y y} / \sigma_{x x}$ for each wafer at the top center of the wafer. (These values vary only slightly with $\sigma_{x x}$.) The ABAQUS model predicts that $\tau_{x y}$ is somewhat smaller than $\sigma_{y y}$, and that the stress components out of the plane ( $z$ direction) are very small, corresponding to a state of plane stress.

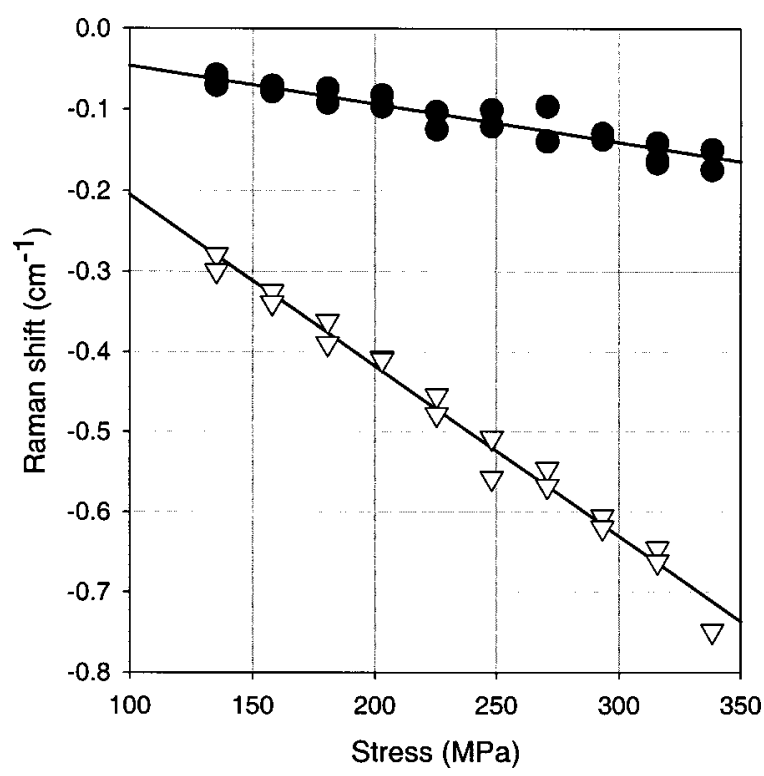

FIG. 4. Experimental data showing how the Raman line center varies with stress $\sigma_{x x}$ (calculated with the ABAQUS model) for two different Polaroid analyzer angles (measured from the laser polarization). Solid dots, $0^{\circ}$; open triangles, $90^{\circ}$. The Raman sensitivity (used as the ordinate in Figs. 6-8) is defined to be the slope of these lines. These data show that the Raman line center position is much more sensitive to stress when the analyzer angle is at $90^{\circ}$ than when it is at $0^{\circ}$.

Our measurements of the Raman sensitivity (variation of the line center position with $\sigma_{x x}$ ) for wafers \#1 and \#2 are shown in Fig. 5. The measured sensitivities (slopes) are $-2.15 \pm 0.1 \mathrm{~cm}^{-1} / \mathrm{GPa}$ for wafer \#1 and $-1.9 \pm 0.1 \mathrm{~cm}^{-1} / \mathrm{GPa}$ for wafer \#2 $(1 \sigma)$. These values are in reasonably good agreement with the value of $-2.0 \mathrm{~cm}^{-1} / \mathrm{GPa}$ predicted for both wafers (shown as solid lines in the figures) using the PDPs of Chandrasekhar et al. ${ }^{17}\left(p / \omega_{0}^{2}=-1.43, q / \omega_{0}^{2}=-1.89\right.$, $\left.r / \omega_{0}^{2}=-0.59\right)$ and using the Table I values for $\sigma_{y y} / \sigma_{x x}$. The agreement is worse if we use the PDPs determined by Anastassakis et al. ${ }^{18}\left(p / \omega_{0}^{2}=-1.85, q / \omega_{0}^{2}=-2.31, r / \omega_{0}^{2}=-0.71\right)$, which predict a sensitivity of $-2.4 \mathrm{~cm}^{-1} / \mathrm{GPa}$ for both wafers. Because only one of the three Raman components is allowed for a normal incidence laser beam on a (100)oriented crystal, rotating the analyzer has no effect on the measured line center, and this strategy cannot be applied. ${ }^{14}$ That is, we cannot determine $\sigma_{y y}$ and $\sigma_{x x}$ independently.

Figure 6(a) shows the sensitivity for wafer \#3 as a function of the analyzer angle, measured from the laser polarization. The maximum measured frequency shift was about $0.7 \mathrm{~cm}^{-1}$, and the sensitivity is calculated by dividing the measured Raman shift for each angle by $\sigma_{x x}$ (calculated by ABAQUS). Each point shown is the average of up to four experimental measurements; a typical error bar representing one standard deviation is shown. The data are bracketed by a pair of curves in the figure, which show the predictions using the PDPs of Chandrasekhar et al. ${ }^{17}$ and assuming either $\sigma_{y y} / \sigma_{x x}=0$ (solid curve) or $\sigma_{y y} / \sigma_{x x}=0.1 \quad$ (long-dashed curve). Thus, our measurements are in good agreement with the ABAQUS result that $\sigma_{y y} / \sigma_{x x}=0.05$. In contrast, Fig. 6(b) shows that we get relatively poor agreement if we use the PDPs of Anastassakis et al. ${ }^{18}$

Figure 7 shows the sensitivity versus the angle for wafer 

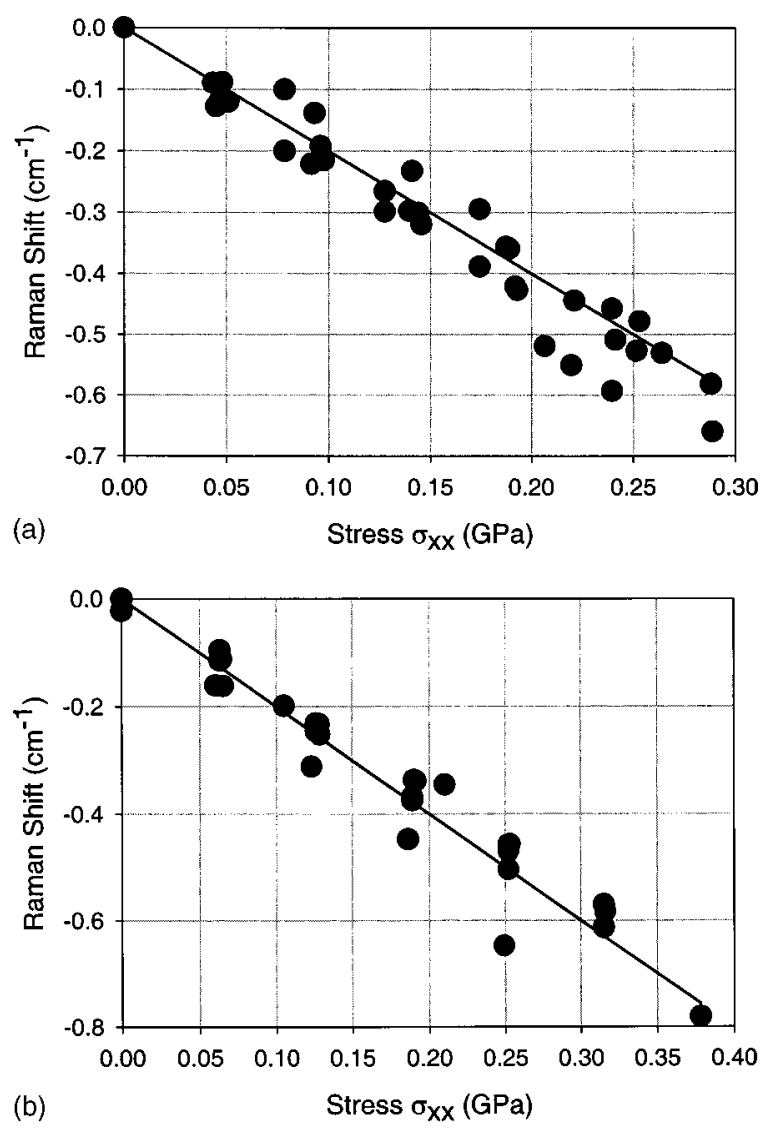

FIG. 5. Variation of the Raman line center with stress for a (001)-oriented wafer. The solid lines show the predicted dependence assuming the PDPs of Ref. 17. (a) Sample \#1, strain along $\langle 100\rangle$. (b) Sample \#2, strain along $\langle 1-10\rangle$.

\#4. Because of a much lower signal level, the scatter is considerably greater than in wafer \#3, as can be seen by comparing the error bars in Figs. 6(a) and 7(a). Using the PDPs of Chandrasekhar et al. ${ }^{17}$ the measurements are in reasonable agreement with the ABAQUS result that $\sigma_{y y} / \sigma_{x x}=0.04$, given the size of the error bars. Here, the long-dashed curve is for $\sigma_{y y} / \sigma_{x x}=0.2$.

The calculated curves shown in Figs. 6 and 7 are nearly independent of the presence of shear stress, so that $\tau_{x y}$ cannot be determined from these measurements. $\tau_{x y}$ can, however, be determined for wafers \#3 and \#4 by rotating the bending device so that the laser polarization is parallel, rather than perpendicular, to $\sigma_{x x}$. Figure 8 shows the sensitivity versus the angle results for wafer \#3, but with the laser oriented along $\langle[1-10]\rangle$. Our measurements show that $0 \leqslant \tau_{x y} / \sigma_{x x}$ $\leqslant 0.1$, consistent with the ABAQUS result that $\tau_{x y} / \sigma_{x x}$ $=0.025$.

In order to use this strategy to determine an unknown state of stress for wafer \#3, for example, the first experiment would use the orientation given in Table I, with the laser polarization perpendicular to $\sigma_{x x}$. Because the shapes of the sensitivity curves in Fig. 6 depend on $\sigma_{y y} / \sigma_{x x}$, a determination of the ratio of the Raman shifts at, for example, $0^{\circ}$ and $90^{\circ}$ determines $\sigma_{y y} / \sigma_{x x}$. The absolute Raman shift at either angle then determines $\sigma_{x x}$ and $\sigma_{y y}$ separately. Knowing these values, we would then rotate the bending device $90^{\circ}$ so that the laser polarization is parallel to $\sigma_{x x}$. Measurements in this
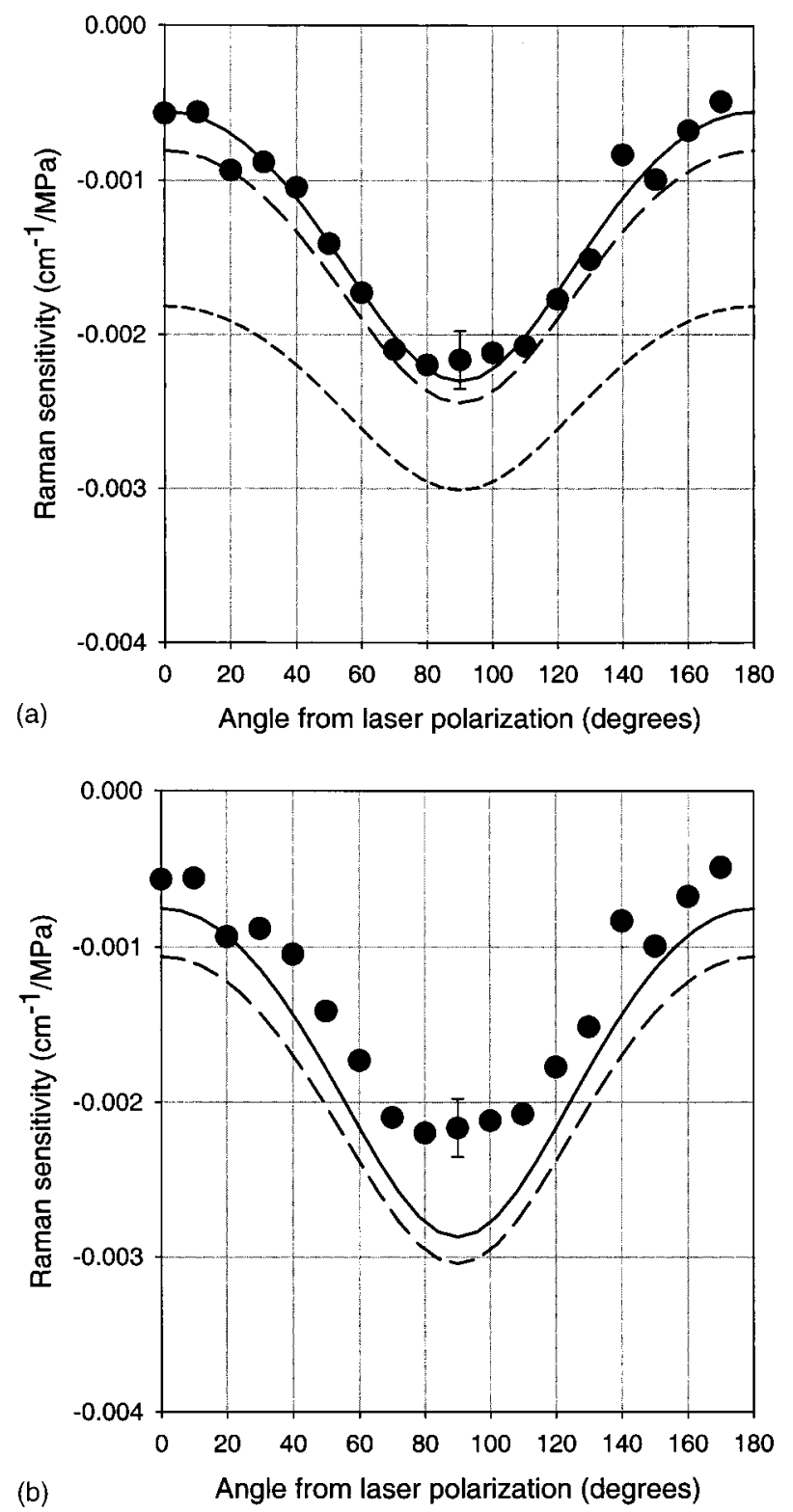

FIG. 6. Raman sensitivity $\left(\mathrm{cm}^{-1} / \mathrm{MPa}\right)$ as a function of the analyzer angle for wafer \#3. (a) Data compared against the prediction using the PDPs of Ref. 17 and (b) data compared against the prediction using the PDPs of Ref. 18. Solid lines, $\sigma_{y y}=0$; long-dashed lines, $\sigma_{y y}=0.1 \sigma_{x x}$; and short-dashed lines, $\sigma_{y y}=0.5 \sigma_{x x}$. $\tau_{x y}$ is assumed to be zero, but the calculated curves are only slightly sensitive to $\tau_{x y}$.

configuration allow $\tau_{x y}$ to be determined from the relative Raman shifts at, say, $60^{\circ}$ and $120^{\circ}$, as can be seen in Fig. 8 .

Because our bending apparatus always gives $\sigma_{x x}$ $\gg \sigma_{y y}>\tau_{x y}$, the Raman strategy has not been tested over a wide range of possible stress states. This will be addressed in a future work. Whether or not the effects of shear and normal stresses can so readily be separated for other crystal orientations will also be examined in future work.

The agreement shown between the theory and experiment in Figs. 6(a), 7(a), and 8 lends support to the strategy proposed by Narayanan et al., ${ }^{14}$ to the accuracy of the ABAQUS model, and to the validity of the PDPs of Chandrasekhar et $a l .{ }^{17}$ But its success is also critically dependent on our ability to measure the very small changes in the Raman line position. Figure 4 indicates that changes as small as 

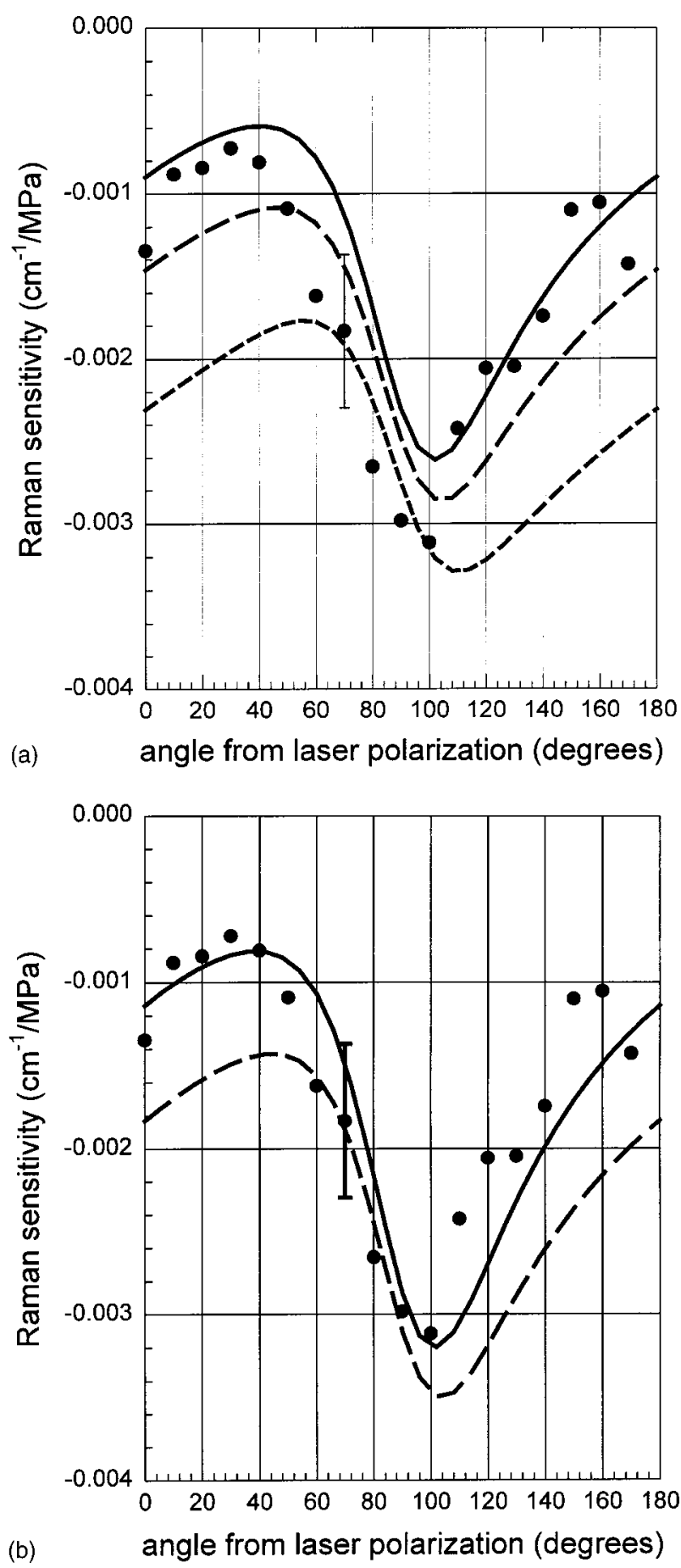

FIG. 7. Raman sensitivity $\left(\mathrm{cm}^{-1} / \mathrm{MPa}\right)$ as a function of analyzer angle for wafer \#4. (a) Data compared against the prediction using the PDPs of Ref. 17 and (b) data compared against the prediction using the PDPs of Ref. 18. Solid lines, $\sigma_{y y}=0$; long-dashed lines, $\sigma_{y y}=0.2 \sigma_{x x}$; and short-dashed lines, $\sigma_{y y}=0.5 \sigma_{x x} . \tau_{x y}$ is assumed to be zero, but the calculated curves are only slightly sensitive to $\tau_{x y}$.

0.02 or $0.03 \mathrm{~cm}^{-1}$ can indeed be detected, consistent with our estimate for the standard error. In turn, this requires that we be able to fit our line shapes accurately and reproducibly with a single functional form, such as that given in Eq. (1), and that Eq. (3) remains valid. Narayanan et al. demonstrated analytically that Eq. (3) is valid for very small splittings. ${ }^{14}$ In the present experiments, the line splittings ranged up to $0.5 \mathrm{~cm}^{-1}$ with a negligible effect on the experi-

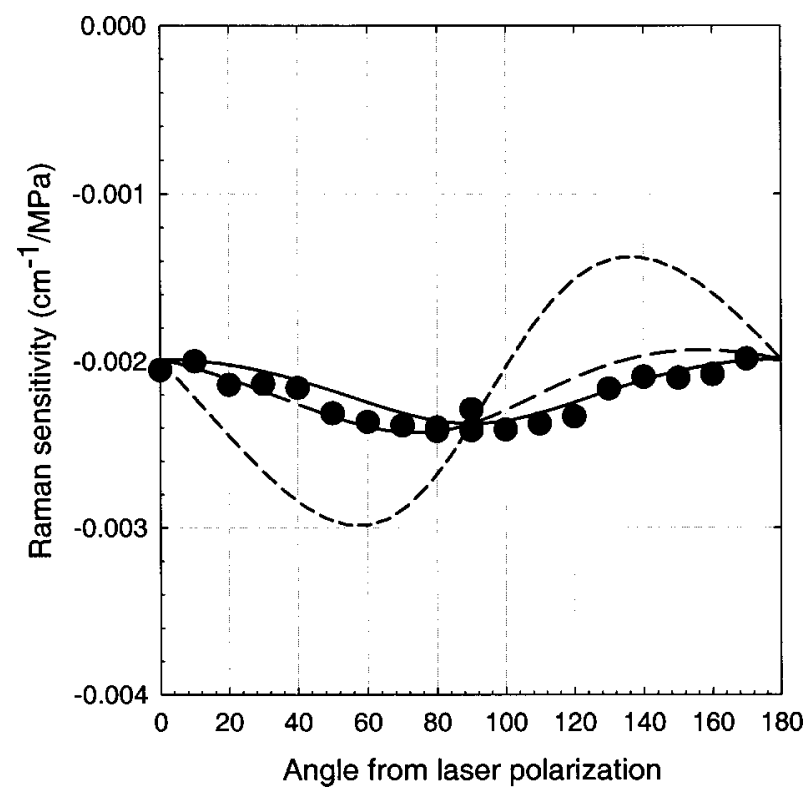

FIG. 8. Raman sensitivity for wafer \#3 but with the laser polarized along the $x$ direction. Solid line, $\tau_{x y}=0$; long-dashed line, $\tau_{x y} / \sigma_{x x}=0.1$; and shortdashed line, $\tau_{x y} / \sigma_{x x}=0.5$. PDPs of Ref. 17 are used.

mental linewidth as determined using Eq. (1). Given the agreement between the theory and experiment found in the present work, we endorse the use of Eq. (3) for splittings as large as $10 \%-15 \%$ of the overall measured linewidth.

We have compared our experimental results to the predictions made using two sets of phonon deformation potentials, and we found better agreement with the results of Chandrasekhar et al. ${ }^{17}$ than with those of Anastassakis et $a l .{ }^{18}$ These two experiments differed in that the latter measured stress through the volume of a silicon sample, while the former measured stress only at the surface. Although there are reasons why volume experiments might be preferred, ${ }^{18}$ the volume experiments had to be made at $110 \mathrm{~K}$, while the surface experiments were made at room temperature. The fact that the PDPs derived from the lowtemperature measurements give consistently poorer fits to our (room temperature) data suggests that the temperature dependence of the elastic properties of silicon may be too important to ignore.

\section{SUMMARY}

1. An ABAQUS model of our bending device was developed that gives strain predictions in good agreement with the experiment.

2. In spite of the modest spectral resolution, our high signal-to-noise ratios allow Raman line center positions to be determined with a reproducibility of about $0.02 \mathrm{~cm}^{-1}$. Such a high precision is required in order to carry out this work.

3. The strategy proposed by Narayanan et al. ${ }^{14}$ for measuring the normal and shear in-plane stress components on the surface of a silicon wafer was validated for the (111)- and (110)-oriented silicon wafers. The strategy cannot be used for the (001)-oriented silicon wafers. 
4. The major stress component $\sigma_{x x}$ was measured with an accuracy of $\pm 10 \%$. The minor stress components $\sigma_{y y}$ and $\tau_{x y}$ were measured with an accuracy of $\pm 0.1 \sigma_{x x}$ or $\pm 0.2 \sigma_{x x}$, depending on the strength of the Raman signal. The latter components were measured to be close to zero, in agreement with the predictions of the finite element model.

5. The phonon deformation potentials of Chandrasekhar et $a l .{ }^{17}$ give better agreement with our data than those of Anastassakis et al. ${ }^{18}$

\section{ACKNOWLEDGMENTS}

The authors gratefully acknowledge the help of Dr. Matti Maricq, Dr. William Donlon, Gary Frank, and Dr. Dairene Uy from Ford. The authors also thank Dr. John Allison from Ford for his invaluable advice and support.

${ }^{1}$ Q. Wang, C. H. Caceres, and J. Griffiths, Metall. Mater. Trans. A 34, 2901 (2003).
${ }^{2}$ T. Clyne, Mater. Sci. Eng., A 122, 183 (1989).

${ }^{3}$ J. D. Eshelby, Proc. R. Soc. London, Ser. A 241, 376 (1957).

${ }^{4}$ T. Mori and K. Tanaka, Acta Metall. 21, 571 (1973).

${ }^{5}$ D. Wilkinson, E. Maire, and J. Embury, Mater. Sci. Eng., A 233, 145 (1997).

${ }^{6}$ D. Wilkinson, W. Pompe, and M. Oeschner, Prog. Mater. Sci. 46, 379 (2001).

${ }^{7}$ Z. Li and R. Bradt, J. Am. Ceram. Soc. 72, 70 (1989).

${ }^{8}$ I. De Wolf, H. Maes, and S. K. Jones, J. Appl. Phys. 79, 7148 (1996).

${ }^{9}$ E. Anastassakis, J. Appl. Phys. 86, 249 (1999).

${ }^{10}$ M. Cazayous et al., J. Appl. Phys. 91, 6772 (2002).

${ }^{11}$ I. De Wolf, M. Ignat, G. Pozza, L. Maniguet, and H. Maes, J. Appl. Phys. 85, 6477 (1999).

${ }^{12}$ G. Loechelt, N. Cave, and J. Menendez, J. Appl. Phys. 86, 6164 (1999).

${ }^{13}$ E. Bonera, M. Fanciulli, and D. Batchelder, J. Appl. Phys. 94, 2729 (2003).

${ }^{14}$ S. Narayanan, S. Kalidindi, and L. Schadler, J. Appl. Phys. 82, 2595 (1997).

${ }^{15}$ J. Brockenbrough and F. Zok, Acta Metall. Mater. 43, 11 (1994).

${ }^{16}$ D. E. Aspnes and A. A. Studna, Phys. Rev. B 27, 985 (1983).

${ }^{17}$ M. Chandrasekhar, J. Renucci, and M. Cardona, Phys. Rev. B 17, 1623 (1978).

${ }^{18}$ E. Anastassakis, A. Cantarero, and M. Cardona, Phys. Rev. B 41, 7529 (1990). 